\title{
Preparation of Strontium Titanate Fine Powder by Thermal Decomposition of Strontium-Titanyl Oxalate
}

\author{
J. Oblakowski and S. Komornicki \\ Academy of Mining and Metallurgy, Department of Materials Science and Ceramics \\ al. Mickiewicza 30, 30-059 Krakow, Poland
}

\section{CONTENTS}

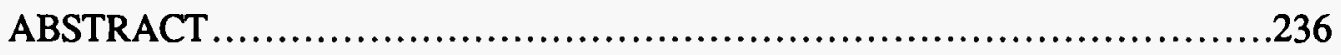

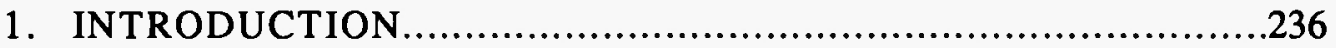

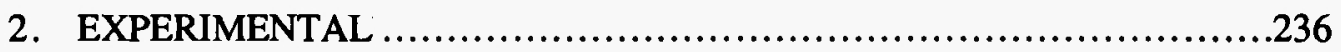

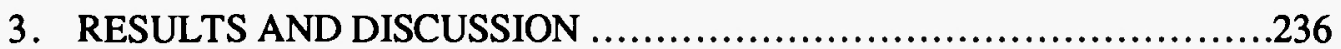

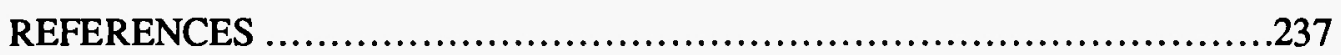




\begin{abstract}
Preparation of strontium titanate fine powder by precipitation and thermal decomposition of strontium titanyl oxalate is described. The mechanism of decomposition was studied and three intermediate stages were found. The final reaction product has regular grains with diameters less than $1 \mu \mathrm{m}$ and a specific surface of about $3.4 \mathrm{~m}^{2} \mathrm{~g}^{-1}$. This powder is suitable for electronic applications as well as for single crystal fabrication.
\end{abstract}

\section{Introduction}

Strontium titanate is widely used in the electronic ceramic industry. The use of single crystals as well as polycrystalline layers of $\mathrm{SrTiO}_{3}$ as substrate to epitaxial growth of high temperature ceramic superconductors has recently been reported; however, its use in fabrication of dielectric boundary layer materials is still an important application. In all these applications, a fine-grained material is necessary to obtain high quality single crystals, thin layers or sintered materials with high density. Strontium titanate can be obtained by solid state reaction of $\mathrm{SrCO}_{3}$ with $\mathrm{TiO}_{2} / 1 /$, by decomposition of some organic precursors $/ 2,3 /$ or by the sol-gel method 14\%. For the very similar $\mathrm{CaTiO}_{3}$, the hydrothermal method was also proposed $15 \%$. The reaction of

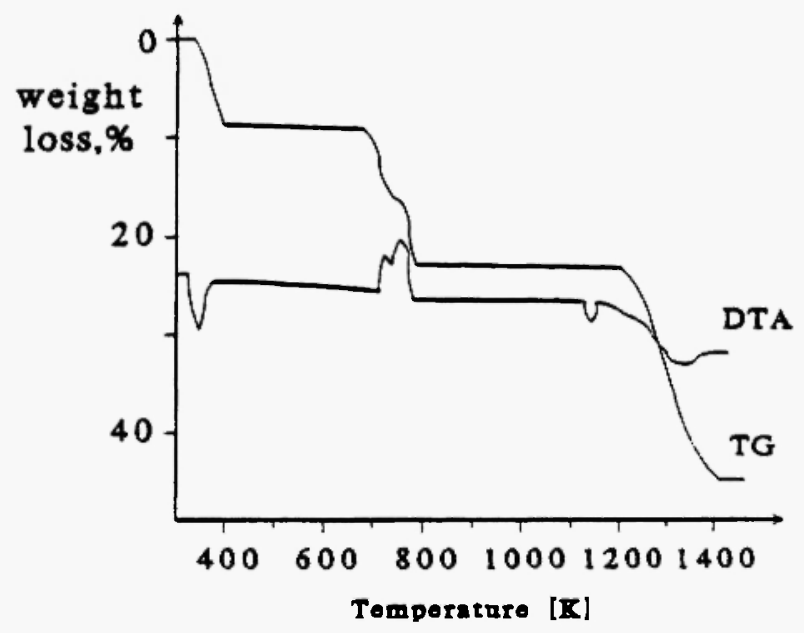

Fig. 1: Thermal decomposition of $(\mathrm{SrTiO})\left(\mathrm{C}_{2} \mathrm{O}_{4}\right)_{2}$ $\cdot \mathrm{nH}_{2} \mathrm{O}$. Heating rate $5 \mathrm{~K} \mathrm{~min}^{-1}$. strontium carbonate with titanium dioxide is simple, but the reaction should be achieved at more than $1400 \mathrm{~K}$; the obtained powder is not very fine and often contains traces of carbonate. Decomposition of the organic precursor should be studied very carefully; however, the pyrolysis of the organic compound yields a fine powder at a much lower temperature.

In this work, we propose a method to obtain fine titanate powder by precipitation of mixed oxalates and their thermal decomposition. This powder is suitable for the preparation of single crystals by the classical Verneuil method; thin and thick layers onto the alumina substrate can also be obtained using it.

\section{Experimental}

$\mathrm{TiCl}_{4}$ and $\mathrm{SrCl}_{2}$ diluted solutions were mixed in the appropriate ratio. Excess of ammonia oxalate was then slowly added to the constantly stirred solution. The precipitate with solution was maintained at $350 \mathrm{~K}$ for 3 hours, then washed by decantation to eliminate $\mathrm{Cl}^{-}$ions, filtered and dried.

This precipitate was decomposed in air and heated for 1 hour at $1370 \mathrm{~K}$. The mechanism of decomposition wâs studied using DTA, TG and DTG coupled equipment (MOM Q-1500 D). X-ray diffraction analyses of precipitate, intermediate products and final titanate were performed. TEM (Philips ZHG-8 Transmission Electron Microscope) was used to estimate grain sizes, grain distribution; specific surface and density were also studied using a Shimadzu SA-CP3 laser grain analyzer.

\section{Results and Discussion}

The curves of DTA and TG of the dried precipitate are presented in Fig. 1. The formula, $(\mathrm{SrTiO})\left(\mathrm{C}_{2} \mathrm{O}_{4}\right)_{2}$. $\mathrm{nH}_{2} \mathrm{O}$, can be attributed to the initial precipitation product; however, this formula would be strictly the same in the case of a two-phase mixture, i.e., $\mathrm{SrC}_{2} \mathrm{O}_{4}$. $\mathrm{TiOC}_{2} \mathrm{O}_{4} \cdot \mathrm{nH}_{2} \mathrm{O}$. The structures as well as the $\mathrm{X}$-ray diffraction patterns of hydrated strontium and titanyl oxalates are unknown; this method cannot settle in these cases. Thermal decomposition of these oxalates invol- 


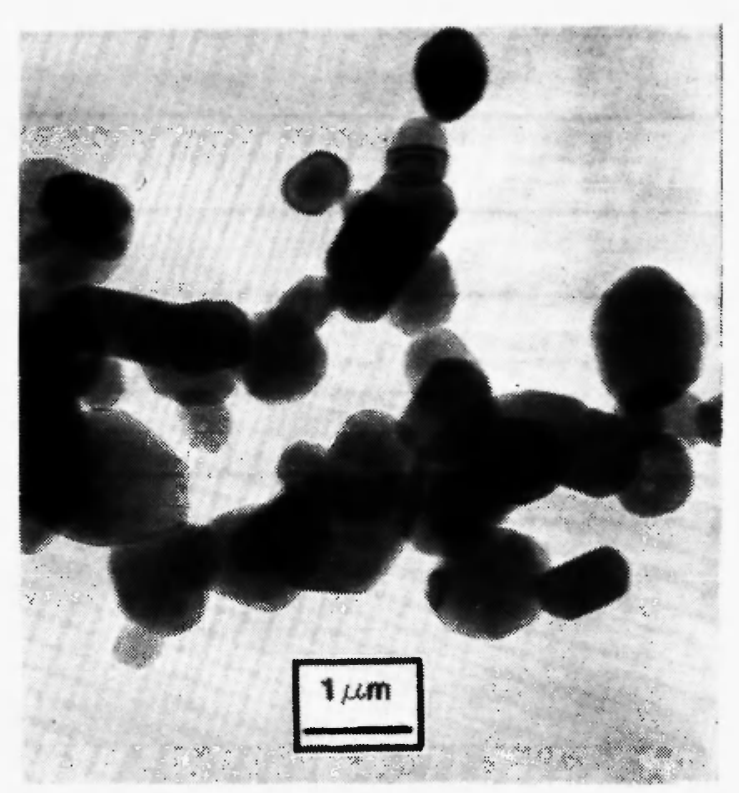

Fig. 2: TEM image of $\mathrm{SrTiO}_{3}$ particles; magnification $10,000 \mathrm{X}$.

ves three distinct stages: i) water and $\mathrm{OH}$ groups elimination below $520 \mathrm{~K}$; ii) transformation of oxalates to carbonates at $670 \mathrm{~K}$; and iii) slow decomposition of carbonates above $1170 \mathrm{~K}$ with the final reaction resulting in $\mathrm{SrTiO}_{3}$ using the following reaction scheme:

$(\mathrm{SrTiO})\left(\mathrm{C}_{2} \mathrm{O}_{4}\right)_{2} \cdot \mathrm{nH}_{2} \mathrm{O} \underset{-\mathrm{j} \hat{2} \mathrm{v} \mathrm{n}}{\stackrel{-n \mathrm{H}_{2} \mathrm{O}}{\longrightarrow}}(\mathrm{SrTiO})\left(\mathrm{C}_{2} \mathrm{O}_{4}\right)_{2}$

$\underset{670-770 \mathrm{~K}}{\stackrel{-2 \mathrm{CO}}{\longrightarrow}}(\mathrm{SrTiO})\left(\mathrm{CO}_{3}\right)_{2} \frac{-2 \mathrm{CO}_{2}}{>1190 \mathrm{~K}} \mathrm{SrTiO}_{3}$

For a two-phase initial composition, the above scheme should be rewritten as two parallel reaction patterns. Some observations of DTA curve yield in precisions:

- two sub-stages in carbon monoxide elimination (two distinct exothermic DTA peaks) confirm separate decomposition of two oxalates; the observed effect is the result of carbon monoxide oxidation in air; and

- phase transformation DTA peak observed about $1190 \mathrm{~K}$. This peak was also observed in $\mathrm{SrCO}_{3}$ during reaction with $\mathrm{TiO}_{2} / 1 /$.

The final product of the reaction is single-phase

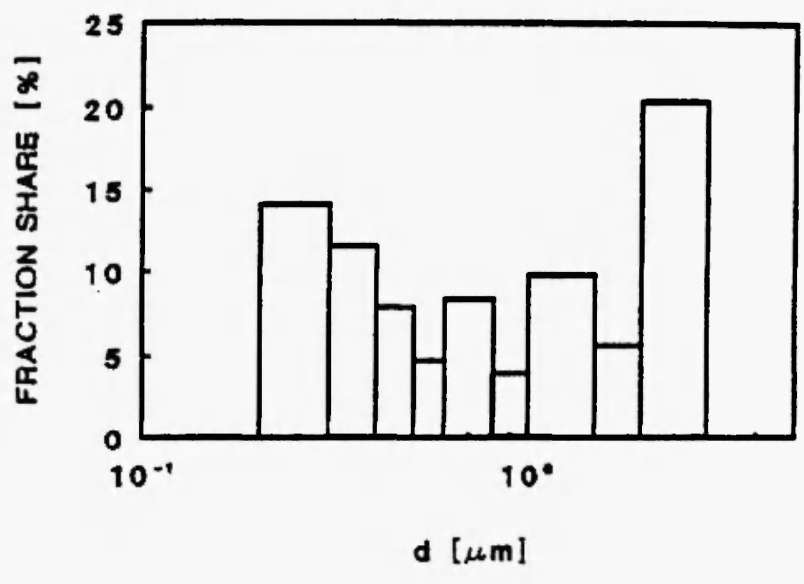

Fig. 3: Granulation of $\mathrm{SrTiO}_{3}$ powder.

$\mathrm{SrTiO}_{3}$, confirmed by its $\mathrm{X}$-ray diffraction pattern. The transmission electron micrograph of the obtained powder is presented in Fig. 2. As one can see in this figure, $\mathrm{SrTiO}_{3}$ particles are regular and their diamęter is less than $1 \mu \mathrm{m}$. In Fig. 3, results of the granulation analysis are presented. The average diameter of grain calculated from these measurements is about $550 \mathrm{~nm}$. The specific surface is estimated to $3.4 \mathrm{~m}^{2} \mathrm{~g}^{-1}$. This last value corroborates TEM observations. The use of this powder in single crystal fabrication by the Verneuil method as well as for screen printing pastes will be reported $/ 6 /$.

The proposed coprecipitation method permits obtaining $\mathrm{SrTiO}_{3}$ single-phase fine powder by thermal decomposition of the precipitate in air. The mechanism of thermal decomposition is simple and carbonates are probably the intermediate phases; however, their final reaction takes place at a relatively low temperature.

\section{References}

1. Tagawa, H. and Igarashi, K., J. Am. Ceram. Soc., 69, 610 (1986).

2. Pecchini, M.P., US Patent 3330697 (1967).

3. Cho, S.G., Johnson, P.F. and Condrate, R.A. Sr., J. Mat. Sci., 25, 4738 (1990).

4. Diaz-Guemes, M.I., Gonzalez Carreno, T., Serna, 
C.J. and Palacios, J.M., J. Mat. Sci., 24, 1011 (1989).

5. Kutty, T.R.N. and Vivekandan, R., Mat. Letters,
5, 79 (1987).

6. Oblakowski, J. and Komornicki, S., in preparation. 